\title{
CORRESPONDENCE
}

\section{Pregnancy outcomes in pulmonary arterial hypertension in the modern management era}

\section{To the Editors:}

We read with interest the important article by JAïs et al. [1] in a recent issue of the European Respiratory Journal discussing management and outcome of pregnancy in pulmonary hypertension patients in the light of recent advanced therapy. We certainly agree that this issue should be revisited in the current era when many new potent medications are available. Yet, even in the current era with optimal management, this condition carries substantial risks and demands careful examination of the data in the literature. Because this condition is rare and only a few case series have been published we wish to contribute our single centre experience with nine pregnancies in seven patients (table 1).

A multidisciplinary team evaluated all patients with frequent echocardiographic surveillance and clinical assessment during pregnancy. All patients were treated with oxygen, if needed, to maintain saturation above $90 \%$, and subcutaneous enoxaparin $1 \mathrm{mg} \cdot \mathrm{kg}^{-1}$ twice daily. We treated all patients except one with intravenous prostacycline during pregnancy and tried to wean them from intravenous medication after delivery [2]. In five cases $(55 \%)$ we used combination therapy with oral medications. We planned elective Caesarean section under general or regional anaesthesia, preferably at 34 weeks of pregnancy if possible. This mode of delivery was also preferred by BONNIN et al. [3]. After delivery all patients remained in an intensive care unit for 1 week of careful monitoring.
Two out of the seven patients died (two out of nine pregnancies) within 2 weeks post-delivery with no fetal mortality in our series.

It is important to note that the fatal outcome rate in the article by JAïs et al. [1] should be four (20\%) out of 20 and should not include patients who underwent induced abortion and did not complete their pregnancy. Maternal mortality rate of $17-33 \%$ is reported by BÉDARD et al. [4] in a systematic review and $22 \%$ in our series. Better outcome is reported by KIELY et al. [5] with no maternal mortality in 10 pregnancies. We think it is imperative to continue with the multicentre registry of JAïs et al. [1] and to add an interventional protocol based on consensus opinion for more consistent recommendations in the future. We agree with the author's cautionary conclusion, not to change the current recommendation to avoid pregnancy in pulmonary hypertension patients. An exceptional group might be those who are diagnosed while pregnant and insist on continuing with pregnancy.

\section{Dror Rosengarten, Leonard C. Blieden and Mordechai R. Kramer}

Institute of Pulmonology, Rabin Medical Center, Beilinson Campus, Petah Tiqwa and Sackler Faculty of Medicine, Tel Aviv University, Tel Aviv, Israel.

Correspondence: M.R. Kramer, Rabin Medical Center, Petah Tikva 49100, Israel. E-mail: kremerm@clalit.org.il

TABLE 1 Pulmonary hypertension: characteristics, management and outcome in pregnancy

\begin{tabular}{|c|c|c|c|c|c|c|c|c|c|c|c|c|c|}
\hline Patient & $\begin{array}{c}\text { Age } \\
\text { yrs }\end{array}$ & Type & NYHA & $\begin{array}{l}\text { mPAP } \\
\text { mmHg }\end{array}$ & $\begin{array}{c}P \text { pcw } \\
\mathrm{mmHg}\end{array}$ & $\mathrm{CO} L \cdot \mathrm{min}^{-1}$ & $\begin{array}{c}\text { PVR } \\
\text { Wood }\end{array}$ & $\begin{array}{c}\text { SPAP } \\
\mathrm{mmHg}\end{array}$ & Medication & Delivery & Anaesthesia & $\begin{array}{c}\text { Outcome: } \\
\text { mother }\end{array}$ & $\begin{array}{c}\text { Outcome: } \\
\text { baby }\end{array}$ \\
\hline 1 & 24 & CTD & $\|$ & NA & NA & NA & NA & 60 & EPO i.v. & CS 32W & GA & Live & Live \\
\hline 2 & 25 & CTD & $\|$ & 28 & 8 & 7.6 & 2.8 & 55 & EPO i.v. + S & CS 34W & GA & Live & Live \\
\hline 3 & 27 & CTD & $\|$ & NA & NA & NA & NA & 65 & EPO i.v.+S & CS 34W & RA & Live & Live \\
\hline 4 & 33 & $\mathrm{CHD}$ & III & 70 & 5 & 5 & 13 & 90 & EPO i.v. & CS 28W & GA & Live & Live \\
\hline 5 & 31 & $\mathrm{CHD}$ & III & 90 & 13 & 2.8 & 27 & 130 & EPO i.v.+S & CS 34W & GA & Live & Live \\
\hline 6 & 26 & $\mathrm{CHD}$ & III & NA & NA & NA & NA & 105 & EPO i.v. + S & CS 34W & GA & Dead & Live \\
\hline 7 & 30 & $\mathrm{CHD}$ & III & 33 & 12 & 4 & 5.2 & 75 & EPO i.v. & CS 32W & GA & Live & Live \\
\hline 8 & 32 & $\mathrm{CHD}$ & III & 50 & 8 & 4.2 & 10 & 70 & ILO inh.+S & CS 34W & $\mathrm{GA}$ & Dead & Live \\
\hline 9 & 31 & IPAH & I & 28 & 12 & 4 & 4 & 80 & TREP i.v. & CS 33W & RA & Live & Live \\
\hline
\end{tabular}

Pregnancies 1-3 are in the same patient. NYHA: New York Heart Association functional classification; mPAP: mean pulmonary artery pressure; Ppcw: pulmonary capillary wedge pressure; CO: cardiac output; PVR: pulmonary vascular resistance; SPAP: maximal systolic pulmonary artery pressure (estimated by echocardiography during pregnancy); CTD: connective tissue disease; CHD: congenital heart disease; IPAH: idiopathic pulmonary arterial hypertension; NA: not available; EPO: epoprostenol, S: sildenafil; ILO: iloprost; TREP: treprostinil; inh.: inhaled; CS: Caesarean section, W: week of pregnancy; GA: general anaesthesia; RA: regional anaesthesia. 
Statement of Interest: None declared.

\section{REFERENCES}

1 Jaiis X, Olsson KM, Barbera JA, et al. Pregnancy outcomes in pulmonary arterial hypertension in the modern management era. Eur Respir J 2012; 40: 881-885.

2 Bendayan D, Hod M, Oron G, et al. Pregnancy outcome in patients with pulmonary arterial hypertension receiving prostacyclin therapy. Obstet Gynecol 2005; 106: 1206-1210.
3 Bonnin M, Mercier FJ, Sitbon O, et al. Severe pulmonary hypertension during pregnancy: mode of delivery and anesthetic management of 15 consecutive cases. Anesthesiology 2005; 102: 1133-1137.

4 Bédard E, Dimopoulos K, Gatzoulis MA. Has there been any progress made on pregnancy outcomes among women with pulmonary arterial hypertension? Eur Heart J 2009; 30: 256-265.

5 Kiely DG, Condliffe R, Webster V, et al. Improved survival in pregnancy and pulmonary hypertension using a multiprofessional approach. BJOG 2010; 117: 565-574.

DOI: $10.1183 / 09031936.00047512$

\section{Chronic bronchitis: an objective diagnosis of exclusion}

\section{To the Editors:}

In their recent, very interesting study, MONTES DE OCA et al. [1] suggested that chronic bronchitis is associated with increased morbidity and poorer general health status in both chronic obstructive pulmonary disease (COPD) and non-COPD subjects. Although the term "chronic bronchitis phenotype" is mentioned in the title, we feel that the differentiation between the phenotype and the actual diagnosis should be highlighted. Chronic bronchitis is a diagnosis of exclusion, while its phenotype could include patients with congestive heart failure (CHF), asthma, tuberculosis, lung cancer, bronchiectasis or other respiratory diseases. All these possible diagnoses were insufficiently investigated in this study. As a result, the overwhelming majority of non-COPD subjects with chronic bronchitis phenotype presented in this study are expected to match one of these diagnoses, with the well-established levels of morbidity and quality of life burden. We believe that the CHF diagnosis should have at least been inquired from all the subjects. In the COPD group, the same confounding factor should have been taken into account.

However, we believe that the prevalence of chronic bronchitis phenotype in COPD patients is underestimated in this study. First, we would like to note the fact that a forced expiratory volume in $1 \mathrm{~s} /$ forced vital capacity ratio of 0.7 is a cut point that lacks a physiological background. As a result, values near 0.7 can neither diagnose nor exclude COPD in the ignorance of the clinical context, especially in the elderly [2]. The significantly lower percentages of respiratory symptoms and of respiratory prescriptions in the nonbronchitic subgroup of COPD stage I patients also suggests a hyperdiagnosis of COPD in subjects without clinical disease. Moreover, the retrospective, subjective questionnaire in clinically stable patients could be misleading, since older people would more easily underestimate symptoms that are "expected for their age", whereas younger patients may overestimate symptoms that restrict their daily activities. This is compatible with the observation that COPD patients with chronic bronchitis show a general trend toward lower age and worse subjective symptoms, which cannot be explained otherwise. Age stratification could be enlightening.

\author{
Alexandros G. Mathioudakis*, Victoria Chatzimavridou- \\ Grigoriadou", Efstathia Evangelopoulou" and \\ Georgios A. Mathioudakis ${ }^{\#}$
}

*Medical Department, Macclesfield District General Hospital, National Health Service, Macclesfield, UK. "Respiratory Dept, General Hospital of Nikaia "St. Panteleimon", Piraeus, Greece.

Correspondence: A.G. Mathioudakis, Medical Dept, Macclesfield District General Hospital, National Health Service, Victoria Road, Macclesfield, SK10 3BL, UK. E-mail: a.mathioudakis@ nhs.net

Statement of Interest: None declared.

\section{REFERENCES}

1 Montes de Oca M, Halbert RJ, Lopez MV, et al. The chronic bronchitis phenotype in subjects with and without COPD: the PLATINO study. Eur Respir J 2012; 40: 28-36.

2 Vaz Fragoso CA, Concato J, McAvay G, et al. Chronic obstructive pulmonary disease in older persons: a comparison of two spirometric definitions. Respir Med 2010; 104: 1189-1196.

DOI: 10.1183/09031936.00107712

From the authors:

We would like to thank A.G. Mathioudakis and co-workers for their interest and comments regarding our recently published article in the July issue of the European Respiratory Journal [1].

A.G. Mathioudakis and co-workers raise a concern regarding the term of "chronic bronchitis phenotype" and the actual diagnosis. They also comment that the phenotype could include patients with tuberculosis, lung cancer, asthma, bronchiectasis and heart failure, and that these diagnoses were not sufficiently investigated in our study. We agree with the position that the phenotype includes patients with other conditions and, in this way, the term was addressed in our study [1]. The self-reported comorbidity (asthma, tuberculosis and lung cancer) of the subjects with 